On the size-dependent elasticity of silicon nanocantilevers: impact of defects

This article has been downloaded from IOPscience. Please scroll down to see the full text article.

2011 J. Phys. D: Appl. Phys. 44072001

(http://iopscience.iop.org/0022-3727/44/7/072001)

View the table of contents for this issue, or go to the journal homepage for more

Download details:

IP Address: 131.180.130.109

The article was downloaded on 10/05/2012 at 14:15

Please note that terms and conditions apply. 


\title{
On the size-dependent elasticity of silicon nanocantilevers: impact of defects
}

\author{
Hamed Sadeghian $^{1,2}$, Hans Goosen ${ }^{1}$, Andre Bossche ${ }^{2}$, Barend Thijsse ${ }^{3}$ \\ and Fred van Keulen ${ }^{1}$ \\ ${ }^{1}$ Structural Optimization and Computational Mechanics (SOCM) group, Department of Precision and \\ Microsystems Engineering, Delft University of Technology, The Netherlands \\ ${ }^{2}$ Electronic Instrumentation Laboratory, Department of Microelectronics, Delft University of \\ Technology, The Netherlands \\ ${ }^{3}$ Virtual Materials Laboratory, Department of Materials Science and Engineering, Delft University of \\ Technology, Mekelweg 2, Delft, The Netherlands \\ E-mail: h.sadeghianmarnani@tudelft.nl and hamed.sadeghian@gmail.com
}

Received 29 November 2010, in final form 10 December 2010

Published 28 January 2011

Online at stacks.iop.org/JPhysD/44/072001

\begin{abstract}
Recent measurements have indicated that the elastic behaviour of silicon nanocantilevers and nanowires is size-dependent. Several theoretical models have been proposed to explain this phenomenon, mainly focused on surface stress effects. However, discrepancies are found between experiments and theories, indicating that there could be other influences in addition to surface effects. One of the important issues, which was experimentally confirmed and has not been considered, is accounting for the fact that experimentally tested nanocantilevers and nanowires are not defect free. In this paper molecular dynamics (MD) is utilized to study the effects of defects on the elasticity of silicon. The effective Young's modulus $\tilde{E}$ of [1 00 ] and $\left[\begin{array}{lll}1 & 1 & 0\end{array}\right]$ oriented silicon nanoplates is extracted in the presence of defects, showing that such defects significantly influence the size-dependent behaviour in $\tilde{E}$. The MD results are compared with the results of continuum theory, showing that continuum theory holds, even for very small defects. Taking into account the surface effects, native oxide layers together with fabrication-induced defects, the experimental measurements can be explained. The studied example involved nanocantilevers, but can be extended to nanowires.
\end{abstract}

(Some figures in this article are in colour only in the electronic version)

Due to advantages of miniaturization, such as smaller size, shorter time response, higher performance and reduced energy requirements, significant research efforts have been directed towards the developments of nanoelectromechanical systems (NEMS). The building blocks of NEMS are mostly nanocantilevers, nanoplates, nanowires and nanotubes. Due to their applications, their mechanical properties are of considerable interest. Recent experimental [1-4] and computational studies, including $a b$ initio and density functional theory (DFT) [5-7], molecular dynamics (MD) [8-11] and modifications to continuum theory [12-15] revealed strong size-dependent mechanical properties as the characteristic dimensions of the structure approached nanometre scale.

Li et al [1] measured the $\tilde{E}$ of [1 110$]$ silicon cantilevers with thicknesses ranging from 300 to $12 \mathrm{~nm}$ using the resonance frequency method. The $\tilde{E}$ showed significant decrease from 167 to $53 \mathrm{GPa}$. Gordon et al [2] performed a new, multipoint bending protocol in an atomic force microscope in order to extract the elastic modulus of vertically aligned Si [1 111 l] nanowires (diameter ranges from 100 to $700 \mathrm{~nm}$ ) in an as-grown state. Zhu et al [4] measured the effective Young's modulus of silicon nanowires with diameters between 15 and $60 \mathrm{~nm}$ and lengths between 1.5 and $4.3 \mu \mathrm{m}$. 


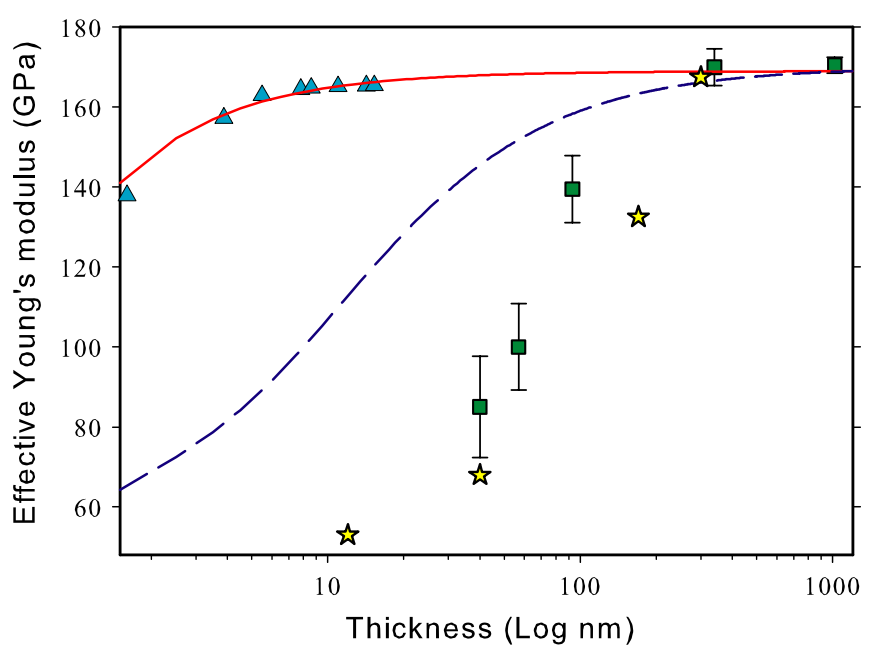

Figure 1. Measured effective Young's modulus of silicon nanocantilevers for different thicknesses (squares [3] and stars [1]). The triangles are the results of direct MD simulation in extensional mode [22]. The solid line shows the prediction of size dependence when considering surface elasticity [15], and the dashed line shows the prediction when additional $2 \mathrm{~nm}$ native oxide layers are taken into account [21].

The nanowires, grown by the vapour-liquid-solid process, were subjected to tensile tests in situ inside a scanning electron microscope.

The size dependence of the effective Young's modulus $\tilde{E}$ has generally been attributed to a number of effects, including surface stress effects $[5,12,15]$, surface contaminations and surface oxidation [16-18], nonlinear bulk elasticity [19], impact of loading method (bending versus extension) [10] and effects of different geometries and surface structures [20]. The succeeding results and discussions have made clear that a substantial gap exists between theoretical predictions and experimental measurements, revealing that although preceding factors influence $\tilde{E}$ to some extent, they alone are insufficient to explain the experimentally observed trends.

As an example, the experimental results of single crystal silicon [1 10 ] nanowires and nanocantilevers reported the observed size dependence at about $100 \mathrm{~nm}$ [1,3,4], while the computational investigations predict this to occur at a characteristic size of less than $10 \mathrm{~nm}[6,8]$. Figure 1 shows a comparison between our recent experimental measurements of the size-dependent bending mode of $\tilde{E}$ of silicon nanocantilevers [3], the experimental results reported in [1] and several theoretical models. The figure shows the effects of surface elasticity in extensional mode of $\tilde{E}$ from MD, a semi-continuum framework [15] without and with a $2 \mathrm{~nm}$ thick native oxide layer. The thickness is shown on a logarithmic scale in order to clearly visualize the difference between theory and experiments. The experimental results showed that the size dependence starts at about $150 \mathrm{~nm}$. The results of semicontinuum approach indicate size effects below $15 \mathrm{~nm}$, i.e. very far from what experimental results showed. Taking the native oxide layer into consideration reduces the difference, yet there is still a considerable gap. Previous work [21] showed that different oxides' properties partially explain the distinctive drops of $\tilde{E}$, but not fully. Therefore, the question remains: why does $\tilde{E}$ decrease at larger thicknesses?

A couple of issues have been raised by researchers in order to reduce the gap between the experiment and the theory $[23,24]$. One of the most important issues, which has not been considered yet, is accounting for the fact that experimentally tested nanostructures are not defect free in contrast to the perfect single crystal structures studied using atomistic simulations [20,23,24]. Recent experimental observations have shown that reducing the thickness of silicon nanocantilevers would not only increase the surfaceto-volume ratio but also the density of fabrication-induced defects [21,25]. Figure 2(a) shows a SEM of so-called $\mathrm{HF}$ defects in a $14 \mathrm{~nm}$ thick silicon device layer. The HF defects are observed as circles with the defect at the centre. Due to convenience, HF etching is often used to test for the existence of these pinhole structures, and the resulting observed defects are called 'HF defects'. The details of the $\mathrm{HF}$ defects were published elsewhere [21]. Using AFM, defects can be observed without HF modification. The AFM image is shown in figure $2(b)$. The white light interferometric picture, shown in figure $2(c)$, shows the appearance of defects on the surface of the cantilever as well. It can be speculated that the HF defects can cause a decrease in $\tilde{E}$ and thus contributes to the discrepancy between experimental measurements and theoretical investigations. In this paper, using MD calculations we investigate the influence of defects on the size-dependent elasticity of silicon nanocantilevers. A recently developed modified embedded atom method (MEAM) potential for silicon [26] was used. Silicon nanoplates were created with initial atomic positions corresponding to the bulk diamond-cubic crystal. The nanoplates were oriented along the $\left[\begin{array}{lll}1 & 0 & 0\end{array}\right]$ and $\left[\begin{array}{lll}1 & 1 & 0\end{array}\right]$ directions. Three different lateral dimensions of nanoplates were studied, large $(40 \times 30$ unit cells), medium $(20 \times 15$ unit cells $)$ and small $(10 \times 8$ unit cells $)$. The thickness was kept as 4 unit cells. Periodic boundary conditions were applied as follows: the plate fills the $x$ and $y$ directions (length and width) completely, but does not fill the $z$ (thickness) direction completely. Thus, there are sufficiently large vacuum regions between the periodic images in the $z$ direction. Consequently, the plates do not interact with each other, so that the top and bottom of the plates represent two free surfaces. Therefore, the system mimics a single nanoplate. The simulation cells were then fully relaxed to a minimum energy state at zero kelvin, while zero pressure was applied in all directions. A time frame of $40 \mathrm{ps}$ was found to be sufficient for the energy minimization. The surfaces display $(2 \times 1)$-type reconstruction along the $\left[\begin{array}{lll}1 & 1 & 0\end{array}\right]$ or $\left[\begin{array}{lll}1 & 1 & 0\end{array}\right]$ directions, consisting of rows of dimers to eliminate one dangling bond per surface atom. At this stage defects with a cylindrical shape of different sizes were created by removing the atoms inside the cylinder. Each nanoplate has one defect at its centre and the radius of the defects varied. Moreover, nanoplates with patterned defects were also studied. The configurations were again fully relaxed to the minimum energy state. Figures $3(a)-(d)$ show the relaxed [ 100$]$ and [ 1110$]$ nanoplates with single defects and patterned defects. Because removing atoms will create free surfaces inside the defect, atoms inside the defect also reconstruct. 

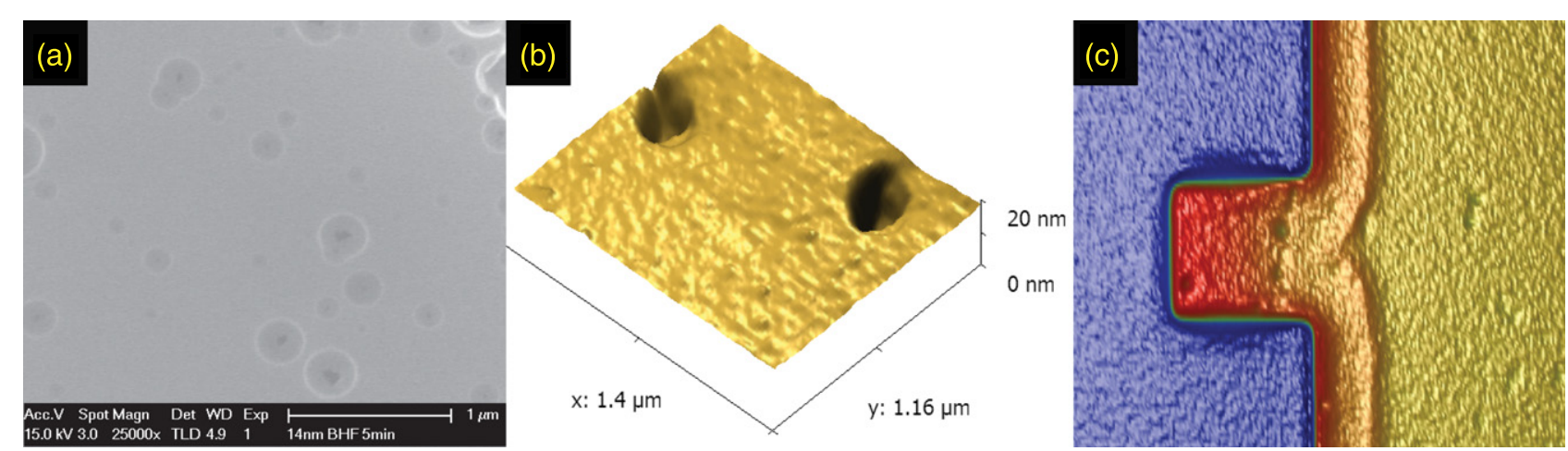

Figure 2. (a) SEM of HF defects in a $14 \mathrm{~nm}$ thick SOI wafer. The BOX layer below the HF defect is etched by HF solution. (b) AFM image of defects without HF modification (shape is caused by AFM probe) and (c) white light interferometric picture of a $40 \mathrm{~nm}$ thick cantilever with defects.

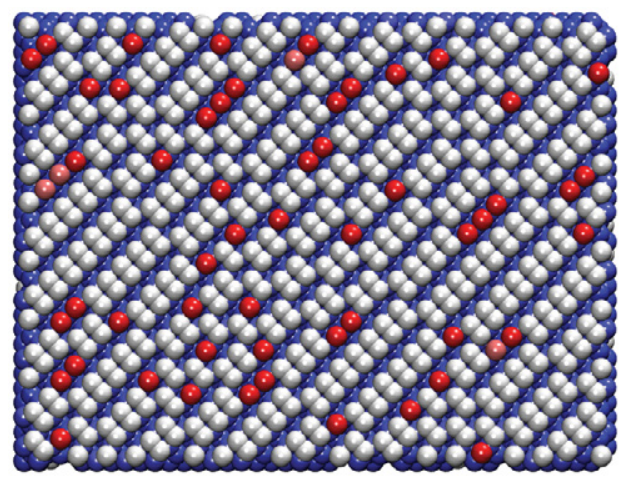

(a)

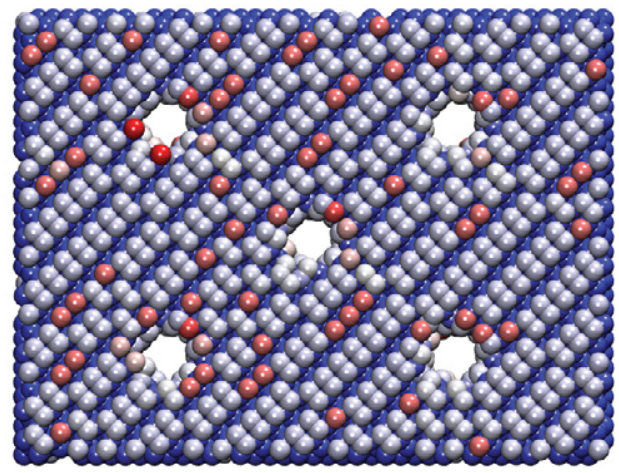

(c)

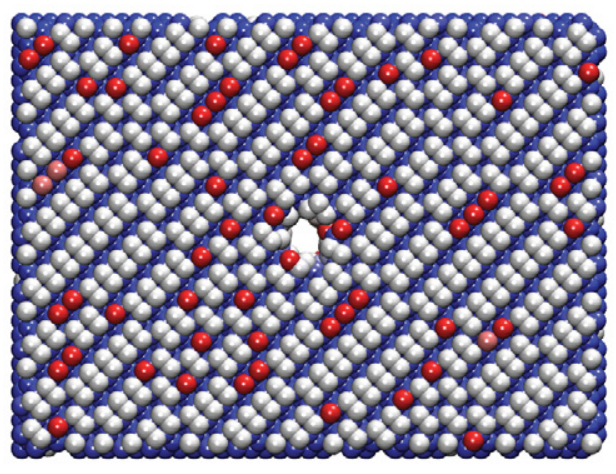

(b)

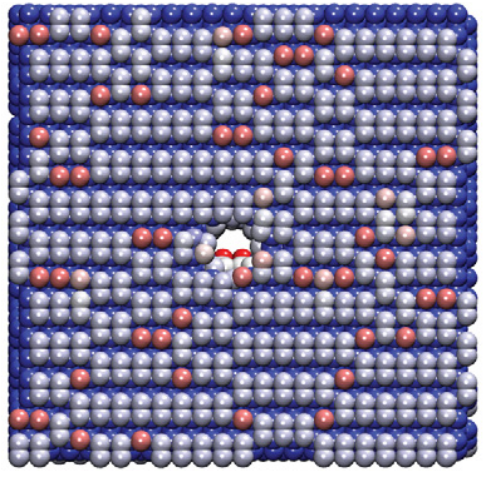

(d)

Figure 3. Snapshots of $(a)$ a fully relaxed defect-free [ 100 l 0 silicon nanoplate, $(b)$ [ 1000 silicon nanoplates with one defect at the centre, $(c)$ patterned defects on [ $\left[\begin{array}{lll}1 & 0 & 0\end{array}\right]$ silicon nanoplate and $(d)\left[\begin{array}{lll}1 & 1 & 0\end{array}\right]$ nanoplate with one defect. Colouring is according to energy. Red colours denotes atoms of higher energy.

After reaching equilibrium configurations, the nanoplates underwent quasi-static tensile loading along the $x$-direction and were again fully relaxed to the local energy minimum following each strain step. The virial stresses [27] were calculated for each level of strain and $\tilde{E}$ was found from the slope of the stress-strain curve. This was repeated for different nanoplates with different sizes of defects. Figure 4 shows $E_{\mathrm{d}} / E_{0}$ versus $A_{\mathrm{d}} / A_{0}$, where $E_{\mathrm{d}}$ and $A_{\mathrm{d}}$ are the effective Young's modulus of the nanoplate with defect and the surface area of defect, and $E_{0}$ and $A_{0}$ are the effective Young's modulus and surface area of the nanoplate without defect. For extremely small defects, the number of atoms removed is not only a function of the defect size, but also of the position of the centre of the defect. When the centre of the defects is moved within the dimensions of a crystal, the number of removed atoms is changed, consequently the effective Young's modulus is changed (see inset in figure 5). This is the reason that in figure 4 the extremely small defects have seemingly noisy $( \pm 0.01)$ values of $E_{\mathrm{d}} / E_{0}$. Re-plotting figure 4 with respect to the number of removed atoms results in figure 5. $N_{\mathrm{d}}$ is the number of removed atoms, and $N_{\mathrm{t}}$ is the total number of atoms. It is noteworthy that creating the defect did not change the average total energy per atom significantly, 


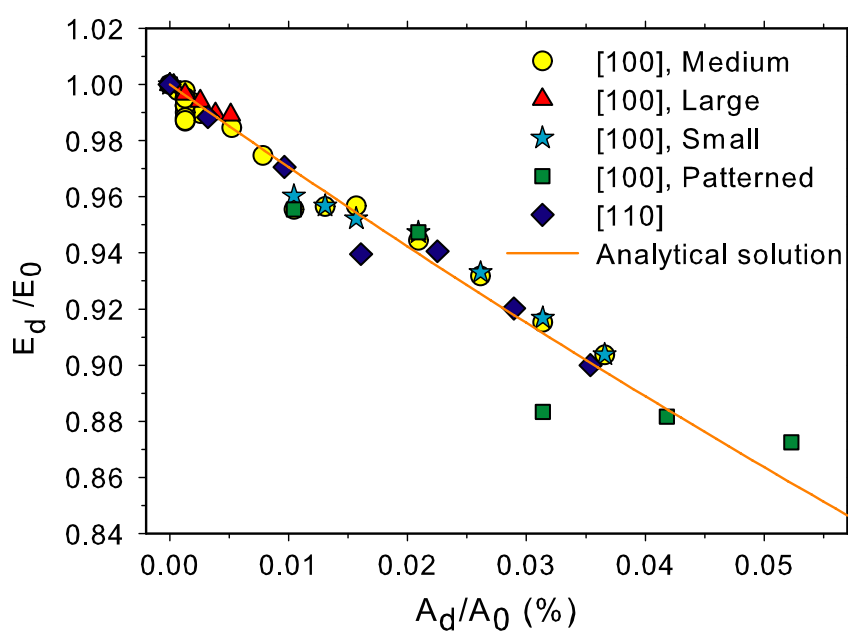

Figure 4. $E_{\mathrm{d}} / E_{0}$ versus $A_{\mathrm{d}} / A_{0}$ for different sizes and directions of nanoplates. The thickness of the nanoplates is 4 unit cells.

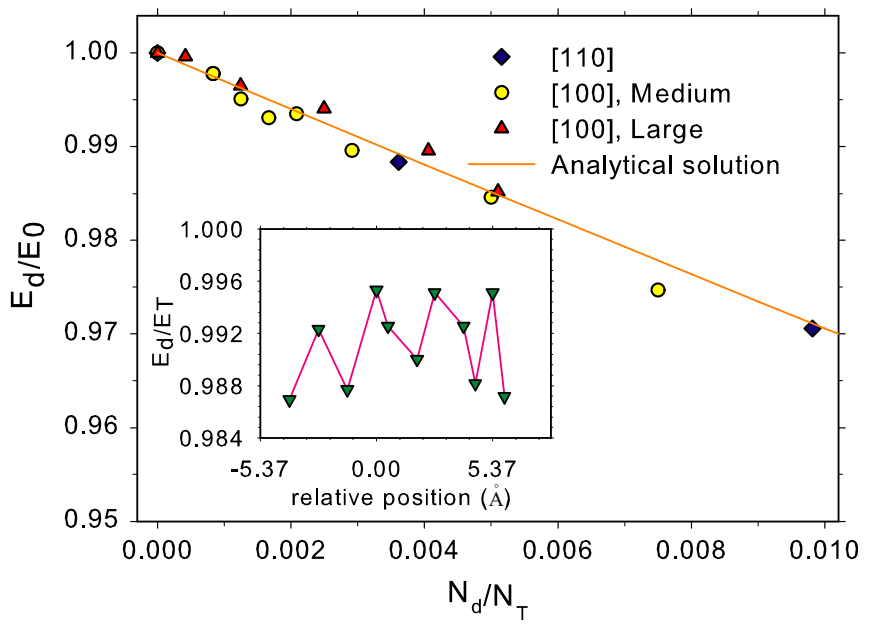

Figure 5. $E_{\mathrm{d}} / E_{0}$ versus $N_{\mathrm{d}} / N_{0}$ for different sizes and directions of nanoplates. The inset shows the $E_{\mathrm{d}} / E_{0}$ as a function of relative position of the centre of the defect to the centre of the nanoplate.

except for the atoms near the surface of the defects, which have higher excess energy. The virial stresses of nanoplates with defects, compared with the uniform virial stress in a nanoplate without defects, are not uniformly distributed, which is due to the stress concentration near the defects. From the changes in the virial stresses $(\triangle \sigma)$ of each individual atom with respect to the stain $(\varepsilon)$, the stiffness contribution of each atom to the $\tilde{E}$ was determined $(\triangle \sigma / \varepsilon)$. This is shown in figure 6 for $\left[\begin{array}{lll}1 & 0 & 0\end{array}\right]$ and figure 7 for [ $\left[\begin{array}{lll}1 & 1 & 0\end{array}\right]$ nanoplates. Substantial changes are found when the size of the defects is increased.

As shown in figures 4 and 5 the MD results were compared with an analytical solution based on continuum theory [28], shown as a solid line. Comparison between the MD results and the analytical solution shows a very good agreement, even for very small defects. Both MD and continuum results indicate that the $\tilde{E}$ linearly decreases with increasing defect volume fraction. Moreover, the MD results confirm an identical decrease in $\tilde{E}$ in different directions, which follows from the analytical solution as well. Since the reduction of the $\tilde{E}$ due to the defects is significant, therefore, it has to be

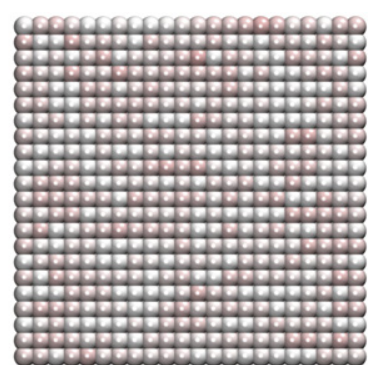

$N_{d} / N_{T}=0.0$

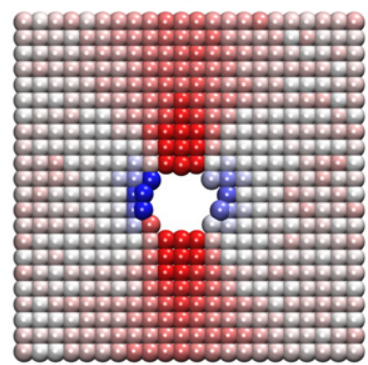

$N_{d} / N_{T}=0.028$

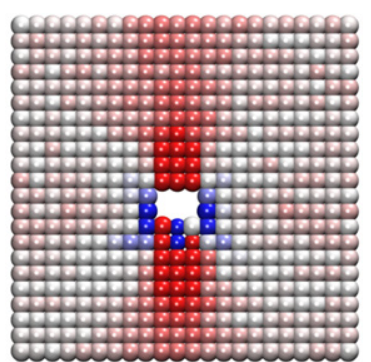

$N_{d} / N_{T}=0.01$

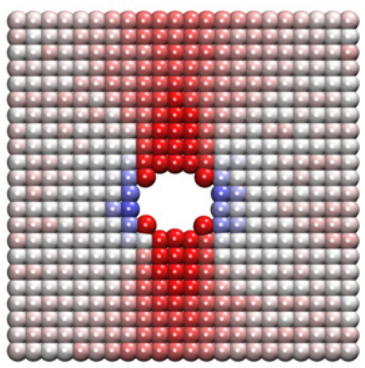

$N_{d} / N_{T}=0.035$
Figure 6. Snapshots of middle layers of silicon [ 1000$]$ nanoplates with different ratios of removed atoms and the total number of atoms. Each individual atom is coloured by its stiffness contribution. Scales are normalized in which 0 corresponds to the colour with lowest contribution to the stiffness and 100 for highest.

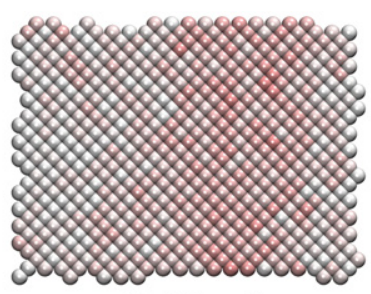

$N_{d} / N_{T}=0$

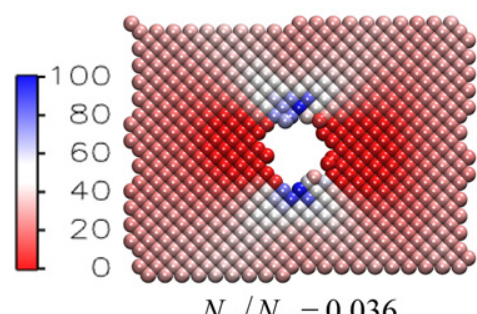

$N_{d} / N_{T}=0.036$

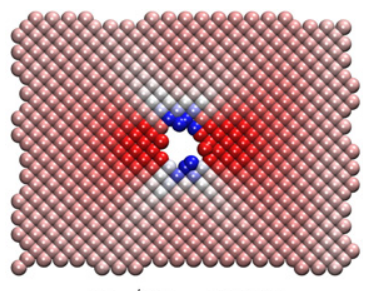

$N_{d} / N_{T}=0.015$

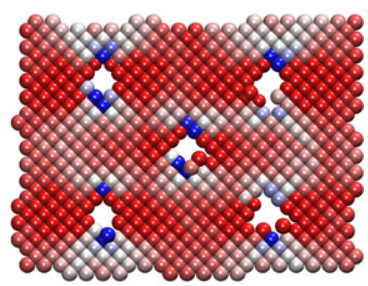

$N_{d} / N_{T}=0.051$
Figure 7. Snapshots of middle layers of silicon [ $\left[\begin{array}{lll}1 & 1 & 0\end{array}\right]$ nanoplates with different ratios of removed atoms and the total number of atoms. Each individual atom is coloured by its stiffness contribution. Scales are normalized in which 0 corresponds to the colour with lowest contribution to the stiffness and 100 for highest.

considered as an additional factor contributing to the decrease in the $\tilde{E}$. Moreover, the native oxide present on a silicon surface would also include the defects present in the silicon. As shown with the analytical solution and the MD results, the effect of defects on $\tilde{E}$ depends strongly on the relative surface ratio of the defects $A_{\mathrm{d}} / A_{0}$ and not on the direction or the material. Therefore, it can be assumed that the effect of defects on the native oxide layers is similar to that of the silicon. By doing so, the effect of defects can now be included in the resultant $\tilde{E}$, affected by surface elasticity and native 


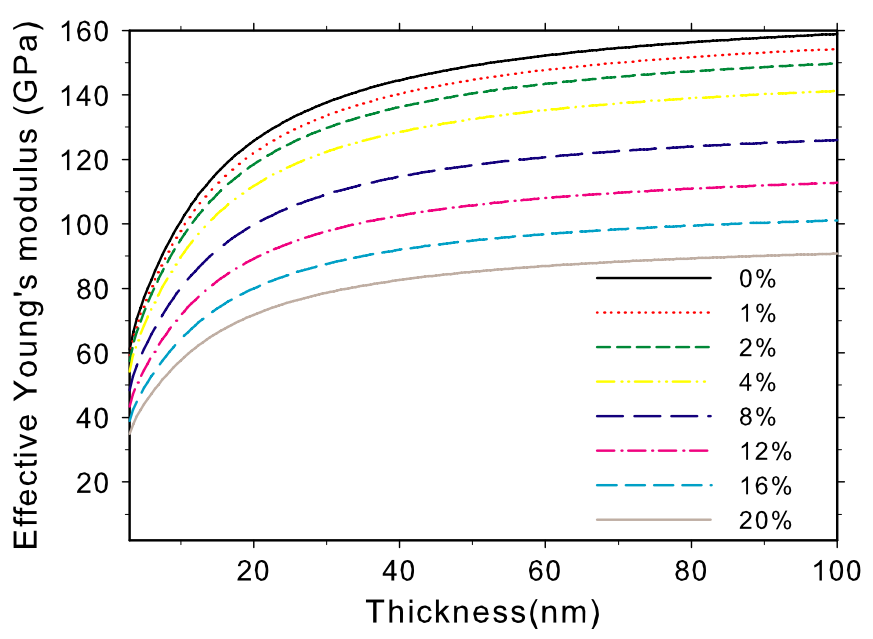

Figure 8. $\tilde{E}$ versus thickness of the cantilever for different defect area fractions.

Table 1. Comparison between experimental $\tilde{E}$ data and those theoretically calculated, taking into account the surface elasticity, native oxide scenarios and fabrication-induced defects. $t_{\mathrm{O} x}$ and $E_{\mathrm{O} x}$ are the thickness and the Young's modulus of native oxide layer.

\begin{tabular}{|c|c|c|c|c|}
\hline \multicolumn{2}{|c|}{$\begin{array}{c}\text { Thickness } \\
\text { (nm) }\end{array}$} & 93 & 57 & 40 \\
\hline \multicolumn{2}{|c|}{$\begin{array}{c}\text { Experimental } \\
\text { data (GPa) }\end{array}$} & $139.5 \pm 8.4$ & $100.1 \pm 10.8$ & $85.2 \pm 12.7$ \\
\hline $\begin{array}{l}t_{\mathrm{O} x} \\
(\mathrm{~nm})\end{array}$ & $\begin{array}{l}E_{\mathrm{O} x} \\
(\mathrm{GPa})\end{array}$ & & & \\
\hline 2 & 75 & $150.91-150.99$ & $119.88-134.16$ & $102.31-114.51$ \\
\hline 5 & 75 & $135.81-142.81$ & $103.53-115.93$ & 84.33-94.73 \\
\hline 2 & 50 & $146.22-154.82$ & $117.84-128.84$ & $97.80-109.40$ \\
\hline 5 & 50 & $130.16-135.16$ & $95.13-107.33$ & $69.08-81.88$ \\
\hline
\end{tabular}

oxide layers. Figure 8 shows the effect of defects of various densities on $\tilde{E}$ as a function of thickness. $0 \%$ corresponds to $\tilde{E}$ of silicon cantilevers with $2 \mathrm{~nm}$ thick native oxide layer and surface elasticity without defect.

The defect density $\left(A_{\mathrm{d}} / A_{0}\right)$, experimentally observed in this work and previous work [25], varies between $0.005 \%$ to $0.12 \%$, depending on the thickness of the silicon device layer. Figure 4 was used to calculate $E_{\mathrm{d}} / E_{0}$ where $E_{0}$ is now the effective Young's modulus including the surface elasticity and the native oxide layer. This was done for different native oxide scenarios, as reported in previous works $[2,21,29,30]$. The results are shown in table 1 . $\tilde{E}$, including the effects of surface elasticity, native oxide layers and fabrication-induced defects, was calculated for the thicknesses that were measured experimentally. The uncertainties in the experimental data are due to the errors of the measurements, reported elsewhere [3]. The variations in the theoretical values are due to the variations in the defect density. It can be seen that taking the fabrication-induced defects into account can explain the observed discrepancy between the experimental measurements and theoretical predictions. As an example, for native oxide of $2 \mathrm{~nm}$ thickness and $E_{\mathrm{O} x}$ of $75 \mathrm{Gpa}$, decreasing the thickness from 93 to 40 nm decreases $\tilde{E}$ from $135.81-142.81$ to 84.3394.73 , similar to what was experimentally observed.

In summary, molecular dynamics calculations have been carried out to determine the effects of fabrication-induced defects on the effective Young's modulus of silicon nanocantilevers. The calculations were motivated by a disparity in the experimental measurements and theoretical investigations of the effective Young's modulus. The conclusion is that the size effect observed in silicon nanocantilevers can be explained by a contribution of surface effects, native oxide layers and fabrication-induced defects. Taking these into account, the gap between the experimental measurements and computational investigation can be closed and a similar trend was observed in the theory. Moreover, the effect of defects is only influenced by the ratio between surface area of the defects and total surface of the nanoplate, similar to what analytical solution based on the continuum theory predicts. Future experimental research on quantifying the size effects on the thickness and the mechanical properties of native oxide layer has to be done. The studied example involved silicon nanocantilevers, but can be extended to nanowires.

\section{Acknowledgment}

This work was financially supported by the Dutch national research program on micro-technology, MicroNed (Project code: IV-C-2).

\section{References}

[1] Li X, Ono T, Wang Y and Esashi M 2003 Ultrathin single-crystalline-silicon cantilever resonators: fabrication technology and significant specimen size effect on young's modulus Appl. Phys. Lett. 83 3081-3

[2] Gordon M J, Baron T, Dhalluin F, Gentile P and Ferret P 2009 Size effects in mechanical deformation and fracture of cantilevered silicon nanowires Nano Lett. 9 525-9

[3] Sadeghian H, Yang C K, Goosen J F L, van der Drift E, Bossche A, French P J and van Keulen F 2009 Characterizing size-dependent effective elastic modulus of silicon nanocantilevers using electrostatic pull-in instability Appl. Phys. Lett. 94221903

[4] Zhu Y, Xu F, Qin Q, Fung W Y and Lu W 2009 Mechanical properties of vapor-liquid-solid synthesized silicon nanowires Nano Lett. 9 3934-9

[5] Umeno Y, Kushima A, Kitamura T, Gumbsch P and Li J 2005 $A b$ initio study of the surface properties and ideal strength of (1 00 ) silicon thin films Phys. Rev. B 72165431

[6] Byeongchan L and Robert E R 2007 First-principles calculation of mechanical properties of $\mathrm{Si}(001)$ nanowires and comparison to nanomechanical theory Phys. Rev. B 75195328

[7] Lee B and Rudd R E 2007 First-principles study of the Young's modulus of Si (0 0 1) nanowires Phys. Rev. B 75041305

[8] Park S, Kim J, Park J, Lee J, Choi Y and Kwon O 2005 Molecular dynamics study on size-dependent elastic properties of silicon nanocantilevers Thin Solid Films 492 285-9

[9] Hyun Woo S, Zhou L G, Hanchen H and Timothy S C 2005 Nanoplate elasticity under surface reconstruction Appl. Phys. Lett. 86151912

[10] McDowell M T, Leach A M and Gall K 2008 Bending and tensile deformation of metallic nanowires Modelling Simul. Mater. Sci. Eng. 16045003

[11] Kang K and Cai W 2007 Brittle and ductile fracture of semiconductor nanowires molecular dynamics simulations Phil. Mag. 87 2169-89 
[12] Miller R E and Shenoy V B 2000 Size-dependent elastic properties of nanosized structural elements Nanotechnology 11 139-47

[13] Wang J, Huang Q-A and Yu H 2008 Size and temperature dependence of young's modulus of a silicon nano-plate J. Phys. D: Appl. Phys. 41165406

[14] Wei G, Shouwen Y and Ganyun H 2006 Finite element characterization of the size-dependent mechanical behaviour in nanosystems Nanotechnology 17 1118-22

[15] Sadeghian H, Goosen J F L, Bossche A and van Keulen F 2009 Surface stress-induced change in overall elastic behavior and self-bending of ultrathin cantilever plates Appl. Phys. Lett. 94231908

[16] Sadeghian H, Yang C K, Gavan K B, Goosen J F L, van der Drift E W J M, van der Zant H S J, French P J, Bossche A and van Keulen F 2009 Effects of surface stress on nanocantilevers e-J. Surf. Sci. Nanotechnol. 7 161-6

[17] Sadeghian H, Yang C K, Gavan K B, Goosen H, van der Drift E, van der Zant H, French P J, Bossche A and van Keulen F 2009 Surface contamination-induced resonance frequency shift of cantilevers Int. Conf. on Nano/Micro Engineered and Molecular Systems (Shenzhen, China) pp 400-3

[18] Sadeghian H, Yang C-K, Gavan K B, Goosen J F L, van der Drift E W J M, van der Zant H S J, Bossche A, French P J and van Keulen F 2010 Some considerations of effects-induced errors in resonant cantilevers with the laser deflection method J. Micromech. Microeng. 20105027

[19] Liang H, Upmanyu M and Huang H 2005 Size-dependent elasticity of nanowires: nonlinear effects Phys. Rev. B 71241403

[20] McDowell M T, Leach A M and Gall K 2008 On the elastic modulus of metallic nanowires Nano Lett. 8 3613-18
[21] Sadeghian H, Yang C-K, Goosen J F L, Bossche A, Staufer U, French P J and van Keulen F 2010 Effects of size and defects on the elasticity of silicon nanocantilevers J. Micromech. Microeng. 20064012

[22] Sadeghian H, Goosen J F L, Bossche A, Thijsse B J and van Keulen F Effects of size and surface on the elasticity of silicon nanoplates: molecular dynamics and semi-continuum approaches Thin Solid Films submitted

[23] Agrawal R and Espinosa H D 2009 Multiscale experiments: state of the art and remaining challenges J. Eng. Mater. Technol. 131041208

[24] Park H S, Cai W, Espinosa H D and Huang H 2009 Mechanics of crystalline nanowires MRS Bull. 34 178-83

[25] Naumova O V, Vohmina E V, Gavrilova T A, Dudchenko N V, Nikolaev D V, Spesivtsev E V and Popov V P 2006 Properties of silicon nanolayers on insulator Mater. Sci. Eng. B 135 238-41

[26] Timonova M, Lee B-J and Thijsse B J 2007 Sputter erosion of $\mathrm{Si}\left(\begin{array}{lll}0 & 0 & 1\end{array}\right)$ using a new silicon MEAM potential and different thermostats Nucl. Instrum. Methods Phys. Res. B 255 195-201

[27] Tsai D H 1979 The virial theorem and stress calculation in molecular dynamics J. Chem. Phys. 70 1375-82

[28] Shevlyakov Y A and Skoblin A A 1993 Relative stiffness of irregularly perforated plates J. Math. Sci. 65 1389-95

[29] Hsin C-L, Mai W, Gu Y, Gao Y, Huang C-T, Liu Y, Chen L-J and Wang Z-L 2008 Elastic properties and buckling of silicon nanowires Adv. Mater. 20 3919-23

[30] Gavrilenko V P, Novikov Y A, Rakov A V and Todua P A 2009 Measurement of thickness of native silicon dioxide with a scanning electron microscope Proc. Instrum. Metrol. Stand. Nanomanuf. III (San Diego, CA) Proc. SPIE 7405740501 